\title{
SPECIALTIES OF HYSTOMORPHOMETRICAL CHANGES IN PLACENTA OF WOMEN WITH EARLY AND LATE PREECLAMPSIA
}

DOI: 10.36740/WLek202001129

\author{
Dmytro G. Konkov, Alina 0. Piskun, Oksana A. Taran, Galyna V. Kostur \\ NATIONAL PIROGOV MEMORIAL MEDICAL UNIVERSITY, VINNYTSIA, UKRAINE
}

\begin{abstract}
The aim: To find out typical pathomorphological differences in placenta of women with early and late preeclampsia.

Materials and methods: Investigation includes 40 placentas from deliveries in women with preeclampsia (main group) and 40 placentas from physiological delivery in somatically healthy women, who had no complications during pregnancy (control group). Placentas in the main group were devided into two sub-groups (20 in each) - with early and late preeclampsia. Specialties of the blood vessels in normal pregnancy were investigated, and their structural transformation with the developement of preeclampsia, according to the appearence of perinatal pathology. Morphometrical data of the blood stream was investigated with the help of eyepiece and program Image Tools 3,6 .

Results: Significant decrease of weight $(p<0,05)$, square and volume of placenta was common to early preeclampsia, comparing to the same characteristics in late Preeclampsia (PE). Specific gravity of villi without vessels, hardened blood vessels, hardened villi and fibrinoid altered vessels was increased statistically significantly $(p<0,05)$ in placenta of women with early $\mathrm{PE}$, comparing to women with late $\mathrm{PE}$. The number of effective blood vessels crossings was determined mostly in late $\mathrm{PE}$, comparing to the early form $(p<0,05)$. Found out significant defferences $(p<0,05)$ in changes of hystovasoarchitecture of placenta in early preeclampsia, according to the number of immature villi and villi with no signs of compensatory angiomatosis.

Conclusions: Increased number of hypoplasia of placenta, breach of effective placental blood stream and significant decrease of compensatory and adaptive changes in placenta are more common to early PE, comparing to late PE.
\end{abstract}

KEY WORDS: late preeclampsia, placenta, gestational endotheliosis

Wiad Lek. 2020;73(1):151-155

\section{INTODUCTION}

Preeclampsia (PE) became the complication of $2-8 \%$ pregnancies in the world, $\mathrm{PE}$ remains one of leading reasons of maternal (50 000 cases annually) and perinatal morbidity and mortality. Perinatal morbidity for this pathology hesitates in limits from 10 to $30 \%$, perinatal morbidity - from 463 to $780 \%$ [1,2]. PE is characterized by increase of systolic blood pressure $\geq 140 \mathrm{mmHg}$ and diastolic $\mathrm{BP} \geq 90 \mathrm{mmHg}$, measuring conducted twice with an interval at least 4 hours. Without regard to the appearence of pathophysiological changes (violation of process of placentation) at the beginning of pregnancy, hypertension and proteinuria, as a rule, show up only in the second half of pregnancy and present in $2-12 \%$ of all cases of pregnancy. The symptoms of PE can embrace the general symptoms of hypertension and / or proteinuria, such as headache, delay of intrauterine growth of the fetus, nausea, vomiting, visual inpairment and edema [3]. In the second and at the beginning of third trimester of pregnancy the treatment of PE is mostly symptomatic, with the aim of normalization of blood pressure and warning of neurological symptoms. In groups with high risk, small decline of risk of development of PE is achived by taking $100 \mathrm{mg}$ of acetylsalicylic acid from 12 weeks of gestation and calcium (1g of elementary calcium in twenty-four hours, from 16 weeks of pregnancy). However, a primary prophylaxis of PE is the a reason of many disscusions and active scientific researches within the framework of that the special attention is payed to the assignment of antiplatelet drugs and vitamin D. Well-proven clinical unefficiency of vitamins-antioxidants $\mathrm{C}$ and $\mathrm{E}$, precursors and donators of oxide of nitrogen, polyunsatured fatty acids for warning the development of hypertension during pregnancy $[3,4]$. The only undoubtedly effective method of treatment of $\mathrm{PE}$ is termination of pregnancy or delivery, although for some women with preeclampsia there can be temporal worsening of condition in postpartum. Grant of help to the women with PE has for an object minimization of the further complications related to pregnancy, prevention of preterm delivery and increase of number of favourable results for mothers and newborn.

In physiological pregnancy the tissue of placenta gets to the uterine arteries of mother that results in the decrease of elasticity of arteries and increases uteroplacental blood flow. In early preeclampsia ( $<34$ weeks) this invasion is insufficient, in the result ischemia of placenta develops and nonoptimal haemodynamic providing of pregnancy. Late $P E$ ( $\geq 34$ weeks) is characterized by greater degree of metabolic violations in the organism of mother, than by an inadequate placental invasion. In both cases as a result development of gestational endotheliopathy, inflammatory maternal immune answer, hypertension and damage of 
vessels in kidneys, liver and brain $[5,6,7]$. The increase of placental invasion and intimacy of contact between mothers and fetus are related with the increase of rates of perinatal growth (but not absolute size) of cerebrum and body of the fetus [7]. Early development of PE has the most unfavorable consequences, as for a mother so for the fetus. Many pathomorphological changes appear in early terms of pregnancy, before clinical symptoms.

\section{THE AIM}

To find out typical pathomorphological and hystomorphometrical differences in placenta of women with early and late preeclampsia.

\section{MATERIALS AND METHODS}

On histological research 40 placentas were taken from physiologycal deliveries from somatically-healthy women, with pregnancy without complications (I group - is a control group) and 40 placentas from deliveries from women with PE (II -main group that was divided into two sub-groups (20 placentas in each sub-group) - with early PE, that developed before 34th week of pregnancy and late $\mathrm{PE}$, that developed after 34 weeks of pregnancy), analysed features of vascular system in norm and features of its structural changes in connection with the development of preeclampsia in different terms.

Pathomorphological research was conducted in extremely early terms after delivery according to the generally accepted methodology. The pieces of placentas were cut out from central, paracentral and regional parts, through all layers of placental tissue, in an amount 8 , by sizes $1,0 \times 0,5 \times 0,5 \mathrm{~cm}$, from an umbilical cord 2 pieces were cut out - in the distance of $2 \mathrm{~cm}$ from the place of attachment of umbilical cord to the placenta and on an opposite side.

Taken material was fixed by $10 \%$ - water solution of neutral formalin not less than 48 hours, it was washed then, dehydrated in the system of polyhydric alcohols, poured in paraffin blocks according to a standard scheme. Prepared semithin cuts, thickness $5-7 \mathrm{mcm}$, were dyed with hematoxylin and eosin and conducted painting after van Gieson, after that conducted the microscopy of the prepared histological preparations by means of light microscope OLIMPUS BX41 increases in 100, 200 and 400 times. Determinations of morphometric parameters of the bloodstream carried out by means of eyepiece, that contains 49 squares, the area of each square is $3,45 \times 10^{-4} \mathrm{MM}^{2}$ at an increase 269,2 and program Image Tools 3,6. The estimation of statistical authenticity of difference of quantitative indexes was executed by means of the program Microsoft Excel. For the further processing of data such statistical methods were used, as getting of mean values and RMS errors, student $t$-test number.

The study was approved by the Ethics Committee of the Vinnytsya Pirogov Memorial National Medical University.

\section{RESULTS}

The middle term of delivery in the control group was $35,1 \pm 2,6$ weeks (women had $32-40$ weeks of pregnancy).
All organometrical parameters - linear sizes, weight and area of maternal surface, were according to the term of gestation. At overview histological research in the cases of normal pregnancy in the general mainly took place involutive and degenerative changes in placental tissues, as moderate fibrosis of stroma and vascular walls of stem and intermediate villi, laying of particles of fibrinoid in a moderate amount at intervillous space and stroma of part of stem villi (signs of physiological maturity of placenta), in some placentas compensatory and adaptive mechanisms took place, such as local compansatory angiomatosis of intermediate and terminal villi $(38,2 \%)$, their budding $(34,3 \%)$ ). In the cases of preterm delivery, the signs of immaturity of placental tissue and compensatory and adaptive mechanisms took place as local compansatory angiomatosis of intermediate and terminal villi $(42,3 \%)$, their budding $(54,8 \%)$.

All organometric parameters - linear sizes, weight and area of maternal surface in part of cases were according to the term of gestation, sometimes placentas had less morphometric values then it should have been, hypoplasia of placentas took place. During overview histological research in the cases of the full-term pregnancy next to general involutory-degenerative and compensatory-adptive changes in placental tissue, took place mildly expressed pathological changes. The signs of immaturity of placental tissue took place in the cases of preterm deliveries, low compensatory-adptive mechanisms, and pathological changes were considerably expressed.

It was set from data of morphological research, that placentas, in II (main) group - I sub-group (early PE)had round and oval forms, however there were placentas of extended irregular shape, three-cornered form, placentas with additional parts and double-particle placentas. Middle weight of placentas in this group was $312,4 \pm 23,2 \mathrm{~g}$, area of maternal surface $172,3 \pm 13,2 \mathrm{~cm}^{2}$, volume $289,8 \pm 8,4 \mathrm{~cm}^{3}$, placenta-fetus coefficient (PFC) was $0,1 \pm 0,02$ (table. 1 ).

The analysis of organometric parameters of placentas in this group showed that in $25 \%$ of cases ( 5 placentas) was found hypoplasia of placenta. Considerable pathological changes were determined on fetal surface. On some placentas the roller of different width took place on the edge, part of placentas had a rim, focal compressions located on the edge, close to the central or in central part of fetal surface. Also the haematomas of different sizes and term, phlebeurysm, took place in some placenta. Unlike in control group, attachment of umbilical cord was mainly regional and thecal with the intermediate and loose type of branching of vessels. Almost in the half of cases hypersinuosity of umbilical cord took place with varicose expansion of veins $(43,2 \%)$, the hypoplasia of Whartons jelly took place on occasion, vessels were located on the edges of umbilical cord, easily collapsed. The transversal crossing of some cords had not round, but extended form, here vessels were located not in triangle form, but in one row and had not round, but extended form.

At the review of maternal surface it was whole, or had defects, with badly expressed cotyledonies, widespread with blood cloats of different term (signs of the partial preterm 
Table 1. Macromorphometric parameters of placentas of women in main and control groups

\begin{tabular}{cccc}
\hline Parameters & $\begin{array}{c}\text { Main group, I subgroup, } \\
\mathbf{n = 2 0}\end{array}$ & $\begin{array}{c}\text { Main group, II subgroup, } \\
\mathbf{n = 2 0}\end{array}$ & $\begin{array}{c}\text { Control group, } \\
\mathbf{n = 4 0}\end{array}$ \\
\hline Weight of placenta, $\mathrm{M}$ & $312,35 \pm 23,14^{*}$ & $405,48 \pm 22,34$ & $472,45 \pm 34,18$ \\
\hline Area, $\mathrm{cm}^{2}$ & $172,26 \pm 13,18^{* *}$ & $194,12 \pm 14,05$ & $268,72 \pm 16,24$ \\
\hline Volume, $\mathrm{cm}^{3}$ & $289,78 \pm 28,4^{* *}$ & $332,68 \pm 29,2$ & $433,38 \pm 31,3$ \\
\hline PFC & $0,1 \pm 0,02$ & $0,12 \pm 0,02$ & $0,18 \pm 0,04$ \\
\hline
\end{tabular}

Notes: * - differences are statistically reliable $(p<0,05)$ in relation to analogical indexes of control group;

** - differences are statistically reliable $(p<0,05)$ in relation to indexes for women with late preeclampsia.

Table 2. Micromorphometric parameters of placentas of women in main and control groups

\begin{tabular}{|c|c|c|c|}
\hline Parameters & $\begin{array}{l}\text { Main group, I subgroup, } \\
\qquad \mathrm{n}=20\end{array}$ & $\begin{array}{l}\text { Main group, Il subgroup, } \\
\qquad n=20\end{array}$ & $\begin{array}{c}\text { Control group, } \\
n=40\end{array}$ \\
\hline $\begin{array}{l}\text { Number of the effective crossings } \\
\text { of vessels }\end{array}$ & $2984516,26 \pm 5512,32^{* *}$ & $3534663,34 \pm 6732,14^{*}$ & $5692318,74 \pm 7886,28$ \\
\hline Specific gravity of avascular villi, \% & $14.85 \pm 0,78^{* *}$ & $10,45 \pm 0,66^{*}$ & $4,52 \pm 0,22$ \\
\hline $\begin{array}{c}\text { Specific gravity of sanguineous } \\
\text { villi, } \%\end{array}$ & $6,54 \pm 0,34^{* *}$ & $16,12 \pm 0,48^{*}$ & $27,36 \pm 1,02$ \\
\hline $\begin{array}{c}\text { Specific gravity of sclerotic } \\
\text { vessels, \% }\end{array}$ & $36,47 \pm 3,4 * *$ & $21,32 \pm 2,4^{*}$ & $11,05 \pm 1,3$ \\
\hline Specific gravity of sclerotic villi, \% & $38,14 \pm 3,3^{* *}$ & $28,32 \pm 3,1^{*}$ & $13,56 \pm 2,2$ \\
\hline $\begin{array}{c}\text { Specific gravity of fibrinoid- } \\
\text { changed villi, } \%\end{array}$ & $18,64 \pm 1,8^{* *}$ & $9,48 \pm 1,1^{*}$ & $4,28 \pm 0,6$ \\
\hline
\end{tabular}

Notes: * - differences are statistically reliable $(p<0,05)$ in relation to analogical indexes of control group;

** - differences are statistically reliable $(p<0,05)$ in relation to indexes for women with late preeclampsia.

placental abruption), hemorragic and ischemic strokes of different localization took place and in different amount, numeral calcinates in decidual plate and in placental tissue. Also the cavities filled with blood, were determined in the layer of placenta tissue.

From data of morphological research of placentas in II sub-group(late PE) of the main group, they had round and oval forms, among that there were placentas of extended irregular-shape, three-cornered form, placentas with additional parts and double-particle placentas. Middle weight of placenta in this group was $405,5 \pm 22,4 \mathrm{~g}$, area of maternal surface $-194,1 \pm 14,1 \mathrm{~cm}^{2}$, volume of $332,7 \pm 9,2 \mathrm{~cm}^{3}$, PFC was $0,12 \pm 0,02$ (table. 1 ) on the average. The analysis of organometric parameters of placenta in this group showed that $10 \%$ cases ( 2 placentas) had hypoplasia of placenta. In some placentas there was a roller of different width on the edge, part of placentas had a rim, focal compressions located on the edge, close to the central or in central part of fetal surface. Also the haematomas of different sizes and term, varicose expansion of vessels, took place in placentas. Attachment of umbilical cord was mainly central or eccentric, in some placentas regional and thecal, with the intermediate and loose type of branching of vessels. Only in some cases hypersinuosity of umbilical cord took place, with varicose expansion of veins (in 3 placentas - 15\%), the hypoplasia of Whartons jelly took place in some cases, vessels were located on the edge of umbilical cord, that easily collapsed. The transversal crossing of some cords had not round, but extended form, here vessels were located not in form of triangle, but in one row and had not round, but extended form.

At the review of maternal surface it was whole, or had defects, however less than it was expressed, comparing to changes in placentas from women with early preeclampsia.

At histological research of placentas of women with preeclampsia of different degree and forms almost in all cases $(89,3 \%)$ violation of maternal and fetal bloodstream was diagnosed, as acute and chronic hemorragic $(26,2 \%)$, and also ischemic $(38,2 \%)$ strokes, widespread hemorrhages at intervillous space $(19,1 \%)$. The presence of small "old" haematomas on the edge of placentas was testified to their partial regional preterm abraption (11,3\%). In some cases $(7,3 \%)$ the thrombosis of intervillous space was determined, mainly in the central zones of placenta. Also there was considerable laying of particles of fibrinoid and fibrin in intervillous space and basale plate, in the places of necrosis of epithelium of villi $(42,1 \%)$, with formation of widespread zones of fibrinoid necrosis. Because of expressed degenerative changes, as a result of considerable disorders of utero-placental bloodstream in placentas, compensatory-adaptive mechanisms developed as focal hyperplasia of terminal villi with their budding $(14,4 \%)$, with focal compensatory angiomatosis (hyperplasia of capillaries) of terminal villi(11,3\%), but comparing to placentas of control group the processes of compensation and adaptation were extremely low.

Also at histological research of placentas such microscopic parameters of microvasculare system, as a number of the 
Table 3. Micromorphometric parameters of placentas of women of main group at early and late preeclampsia

\begin{tabular}{|c|c|c|c|c|}
\hline Pathomorphological changes & $\begin{array}{l}\text { Early preeclampsia, } \\
\qquad n=20, n(\%)\end{array}$ & $\begin{array}{l}\text { Late preeclampsia, } \\
\qquad n=20, n(\%)\end{array}$ & $\mathbf{p}$ & $\mathrm{RR}, \mathbf{9 5} \% \mathrm{Cl}$ \\
\hline Laiyng of fibrinoid at intervillous space & $12(60,0)$ & $8(40,0)$ & 0,21 & $1,5(0,79-2,86)$ \\
\hline Trombosis of vessels of villi tree & $7(35,0)$ & $4(20,0)$ & 0,30 & $1,75(0,61-5,05)$ \\
\hline Trombosis of intervillous space & $4(20,0)$ & $1(5,0)$ & 0,19 & $4,0(0,49-32,73)$ \\
\hline Immature villi & $18(90,0)$ & $5(25,0)$ & 0,001 & $3,6(1,66-7,80)$ \\
\hline Villi with sclerotic, fibrinoid-changed stroma & $7(35,0)$ & $2(10,0)$ & 0,09 & $3,5(0,83-14,83)$ \\
\hline Fresh Hemorrhagic strokes & $8(40,0)$ & $2(10,0)$ & 0,06 & $4,0(0,97-16,55)$ \\
\hline Old Ischemic strokes & $5(25,0)$ & $1(5,0)$ & 0,13 & $5,0(0,64-39,06)$ \\
\hline Laiyngs of big calcinates & $4(20,0)$ & $2(10,0)$ & 0,39 & $2,0(0,41-9,71)$ \\
\hline Villi with signs of compensatory angiomatosis & $4(20,0)$ & $11(55,0)$ & 0,04 & $0,36(0,14-0,95)$ \\
\hline $\begin{array}{l}\text { Ammount of terminal and intermediate villi } \\
\text { with features of budding }\end{array}$ & $3(15,0)$ & $9(45,0)$ & 0,06 & $0,33(0,105-1,05)$ \\
\hline Trombosis of umbilical cord vessels & $6(30,0)$ & $1(5,0)$ & 0,08 & $6,0(0,79-45,42)$ \\
\hline
\end{tabular}

effective crossings of vessels, specific gravity of avascular villi, specific gravity of sanguineous villi, specific gravity of sclerotic vessels, specific gravity of sclerotic villi and specific gravity of fibrinoid-changed villi, were determined.

From results given by us in a table it's evident, that at early $\mathrm{PE}$ there were expressed changes in the vessels of microvasculare system, namely, an amount of the effective crossings of vessels at early PE was almost in 1,5 times less than, at late $\mathrm{PE}$ and 2 times less than, in the placentas of women who had physiologycal pregnancy. More than three times, specific gravity of avascular villi grew in the cases of early PE comparing to normal deliveries and 1,5 times more as compared to late preeclampsia. Specific gravity of sanguineous villi in a control group 4 times higher comparing to a main group, that can testify to the considerably higher index of bloodstream in normal placentas. In the cases of preeclampsia specific gravity of sclerotic villi and specific gravity of sclerotic vessels in villi was three times higher than the same index at physiological gestation. Also the amount of fibrinoid-changed villi was 4 times higher in the cases of perinatal pathology, with a reliable difference between the sub-groups of pathology $(p<0,05)$.

Thus, from pathological processes in placentas from two comparative groups is diagnosed connection of disorders of utero-placental circulation with general dystrophic changes in villi, as a result of violation of microcirculation. It follows, that in placentas at early PE considerably more often there were disorders of utero-placental circulation, such as hemorragic strokes, focal thrombosis and stasis at intervillous space of subbasale and central zones. Dystrophic changes with the sclerosis of stroma of villi and laying of fibrinoid at villi's stroma, and intervillous space with the development of large focal fibrinoid necrosis, was clearly determined at early preeclampsia in moderate and heavy degrees, as a result of violation of microcirculation in villi of all calibers.

At research of histological preparations, such pathological changes were educed in the structure of placentas, as a fall of fibrinoid at intervillous space. At the early PE a similar finding was more evident $(60,0 \%)$ comparing to late preeclampsia (40,0\%), RR 1,5, CI of 95\%, 0,79-2,86, p=0,21 (table. 3). Also we investigated the cases of thrombosis of vessels of villi tree, which one at the early PE had a greater value $-35,0 \%$ cases, and at late PE - 20,0\%, however the difference between indexes that were investigated, also did not have signs of authenticity, RR 1,75, CI of $95 \%, 0,61-5,05, \mathrm{p}=0,30$. The thrombosis of intervillous space took place at early $\mathrm{PE}$ in $20,0 \%$, and at late $-5,0 \%$ cases, RR 4,0, CI of $95 \%, 0,49-32,73, p=0,19$. Immature villi were determined more often in placentas from patients with the cases of early PE (90,0\%), than in late PE( $25,0 \%)$, RR 3,6, CI of $95 \%, 1,66-7,80, p=0,001$.

Next to the changes listed above structural violations, there took place structural changes in villi of different caliber, namely it was presence of fibrinoid-changed and sclerotic stroma, that were 3,5 times higher in the cases of early PE, comparing to late $\mathrm{PE}-35,0 \%$ against $10,0 \%$, RR 3,5 , CI of $95 \%, 0,83-14,83, \mathrm{p}=0,09$.

We determined fresh hemorrhagic and old ischemic strokes in placentas, their specific gravity was also higher at early preeclampsia $-40,0 \%$ and $25,0 \%$ against $10,0 \%$ and $5,0 \%$ at late $\mathrm{PE}$, however marked differences also were not statistically reliable (RR 4,0, CI of $95 \%, 0,97-16,55, \mathrm{p}=0,06$ and RR 5,0, CI of 95\%, 0,64-39,06, $\mathrm{p}=0,13$ ).

Severity of compensatory-adaptive mechanisms in the cases of early preeclampsia was statistically less expressed - the amount of villi with the signs of compensatory angiomatosis here was $20,0 \%$ against $55,0 \%$ at late PE, RR 0,36 , CI of $95 \%, 0,14-0,95, p=0,04$. Amount of intermediate and terminal villi with the signs of budding with syncytial knots at early PE was $15,0 \%$, at late PE - 45,0\% cases, RR 0,33 , CI of $95 \%, 0,105-1,05, p=0,06$. At the same time next to changes in placental tissue pathological changes took place also at the umbilical cord, such as thrombosis of vessels, specific gravity of which at early preeclampsia was $30,0 \%$, at late $-5,0 \%$ cases, RR 6,0, CI of $95 \%, 0,79-45,42, p=0,08$.

\section{DISCUSSION}

Preeclampsia is a multisystem disease of multivariable genesis including imperfect placentation, oxidizing stress, 
autoimmunity, activating of thrombocytes and thrombin, intravascular inflammation, disbalance in angiogenesis, violation of cardiovascular activity of mother and gestational endotheliopathy - as triger of PE. Insufficient invasion of trophoblast can lead to elemination of embryo already in the first weeks of pregnancy, or, in case of progressing of pregnancy, to limitation of growth abilities of placenta, such as her hypoplasia with further development of different obstetric complications [6]. An imperfect placental invasion for certain is related to most cases of early and heavy PE. However, imperfect placentation is less important for development of PE, that shows up later, after 34 weeks. According to literature, comparing to early preeclampsia, at late PE, placenta has considerably low frequency of histological violations, however, maternal factors (for example, metabolic syndrome or chronic hypertension) have a greater meaning. Differences between the early and late stage of PE are also observed in risk factors, maternal vascular reactivity, efficiency of screening and efficiency of prophylaxis.

We found substantial changes of placental histovasoarchitecture, that probably resulted in the decline of perfusion at the early beginning of preeclampsia comparing to physiological pregnancy. Indicated by us corresponds previous ultrasonic research of violations of blood flow in uterus arteries at early preeclampsia [3] and to thistopathological conclusions about the increase of amount of imperfect placentation in women with early PE [7]. In addition, as it shown in previous researches, kids born from mothers with early preeclampsia, had significantly less weight at birth comparing to the babies born from normotensive women in similar gestational age [7], what can indicate on violation of nutritional function of placenta.

The results of our research correspond previous researches, which indicated that late $\mathrm{PE}$ is connected with general hypoperfusion [1,5], while early $\mathrm{PE}$ is connected with hypoperfusion [5]. Brought up researches also showed the increase of cardiac output in combination with the decrease of peripheral resistance in women who developed late preeclampsia [5]. On the other hand, in women who developed early PE, the decrease of cardiac output and increase of peripheral resistance was diagnosed $[1,5]$.

Thus, conducted histomorphometric researches of preparations confirmed dependence of changes of structures of placenta to the form of preeclampsia. For early preeclampsia lowerness of indexes of compensatory-adaptive mechanisms was common, and extremely heavy pathological changes in tissue of placenta that probably resulted in violation of the haemodynamic providing of pregnancy. As the amount of participants in this research is small, it can be, that the differences found can be conditioned by mixed factors.

\section{CONCLUSIONS}

Lower weight ( $\mathrm{p}<0,05)$, area and volume of placenta was common for early preeclampsia comparing to analogical indexes at late PE.
Violation of effective vascular component at early preeclampsia was met, statistically ( $\mathrm{p}<0,05)$ more often, than at the late form of PE.

During comparative micromorphometric research there were found reliable differences $(p<0,05)$ in early preeclampsia - amount of immature villi and villi with the signs of compensatory angiomatosis, than at late preeclampsia.

\section{REFERENCES}

1. Konkov D.G., Piskun A.0. The features of placental angiogenesis in early preeclampsia. Actual questions of modern gynecology and perinatology. 2018:4:25-9.

2. Pia M. Villa, Pekka Marttinen, Jussi Gillberg, A. et al. Cluster analysis to estimate the risk of preeclampsia in the high-risk Prediction and Prevention of Preeclampsia and Intrauterine Growth Restriction (PREDO) study. PLoS One. 2017;12 (3):e0174399.

3. Medved V. I., Zhuk S. I., Konkov D. G., et al. Severe preeclampsia. CALM DOWN - algorithm of actions of medical personnel. Woman's health. 2017;10:28-33.

4. Mifsud W, Sebire NJ. Placental pathology in early-onset and late-onset fetal growth restriction. Fetal Diagn Ther 2014; 36: 117-128.

5. Sohlberg S, Mulic-Lutvica A., Lindgren P., et al. Placental perfusion in normal pregnancy and early and late preeclampsia: A magnetic resonance imaging study. Placenta 2014.;3:202-206.

6. Sotiriadis A, Hernandez-Andrade E, da Silva Costa F. et al. ISUOG Practice Guidelines: role of ultrasound in screening for and follow-up of preeclampsia. Ultrasound Obstet Gynecol 2018.

7. Sulistyowati S. Early and Late Onset Preeclamsia:What did really Matter? J. Gynecol Women's Health. 2017;5(4):1-3.

\section{ORCID and contributionship:}

Dmytro G. Konkov - 0000-0002-9375-7509 A,F

Alina O. Piskun - 0000-0002-1466-716X $X^{B, D}$

Oksana A. Taran - 0000-0002-8808-7539 ${ }^{\circ}$

Galyna V. Kostur - 0000-0002-9420-6696 ${ }^{\mathrm{E}}$

\section{Conflict of interest:}

The Authors declare no conflict of interest

\section{CORRESPONDING AUTHOR Alina 0. Piskun \\ National Pirogov Memorial Medical University \\ Pirogova str. 56, Vinnytsia, Ukraine \\ tel: +380936808258 \\ e-mail:alinapiskun.vn@gmail.com}

Received: 14.04 .2019

Accepted: 01.12.2019
A - Work concept and design, B - Data collection and analysis, C - Responsibility for statistical analysis, D -Writing the article, $\mathbf{E}$-Critical review, $\mathbf{F}$ - Final approval of the article 